A collaboration between

\section{cousins: The Canadian Journal of Public Health and the Canadian Society for Epidemiology and Biostatistics}

We are excited to present a Special Section in this issue of the Journal, which marks a new collaboration between close cousins, the Canadian Journal of Public Health $(\mathrm{CJPH})$ and the Canadian Society for Epidemiology and Biostatistics (CSEB). Included in this Special Section are manuscripts by authors who presented their research at the Biennial CSEB Conference that was held in Toronto, Ontario, June 1-4, 2015. Presenters were invited to submit a manuscript to CJPH based on their submitted abstract. The submitted manuscripts underwent the normal peer-review process, but with an emphasis on engaging CSEB members as reviewers. In addition to these manuscripts, Dr. Nancy Kreiger has provided a written version of her plenary talk entitled "Epidemiology today: Mitigating threats to an ecosystem". This Special Section provides a way to highlight Canadian epidemiologic research within the broader public health community.

In Canada, a journal specific to epidemiology does not exist. Although a topic of discussion for the past 20 years or more, starting a Canadian epidemiology journal is not a viable option at the present time. Upon contrasting the mandates of public health journals with epidemiology journals, we will note more similarities than differences. The CJPH is "dedicated to fostering excellence in public health research, scholarship, policy and practice" and the American Journal of Public Health (AJPH) is "dedicated to publication of original work in research, research methods, and program evaluation in the field of public health". Similar to other epidemiology journals, the American Journal of Epidemiology (AJE) describes itself on its website to be "devoted to the publication of empirical research findings, opinion pieces, and methodological developments in the field of epidemiologic research". Thus, the greatest distinction seems to be "public health research" versus "epidemiologic research". Epidemiology has often been considered the "basic science of public health" and, as such, a subset of public health research. It often has a greater emphasis on upstream rather than applied research, and on methodologic innovations. Ultimately, however, both disciplines have a common goal: to improve population and public health. So, does epidemiology have a "home" in CJPH? Absolutely.

The theme of the Biennial CSEB Conference was "Paradigms to Pragmatism: Epidemiology and Biostatistics for the Changing World". It reflected the vision for a professional society that has reached maturity and celebrates an exceptionally rich landscape of substantive and methodological contributions in Canada. The eight contributed manuscripts in the Special Section in this issue reflect the diversity of epidemiologic research that was showcased in Toronto last June. Two of the papers focus on the distribution of health outcomes (brain cancer and sedentary time), two papers focus on identifying determinants and etiology of health

\section{Une collaboration entre cousins :} La Revue canadienne de santé publique et la Société canadienne d'épidémiologie et
de biostatistiques

Nous sommes impatients de présenter dans ce numéro de la Revue une section spéciale qui marque une nouvelle collaboration entre deux proches cousins : la Revue canadienne de santé publique (RCSP) et la Société canadienne d'épidémiologie et de biostatistiques (SCEB). On trouve dans cette section spéciale les manuscrits d'auteurs qui ont présenté leurs recherches à la Conférence biennale de la SCEB tenue à Toronto (Ontario) du $1^{\mathrm{er}}$ au 4 juin 2015. Les orateurs et oratrices ont été invités à soumettre un manuscrit à la RCSP sur la base du résumé soumis à la conférence. Les manuscrits ont fait l'objet du processus normal d'évaluation par les pairs, mais en insistant sur le recrutement de membres de la SCEB comme évaluateurs. En plus de ces manuscrits, $\mathrm{M}^{\text {me }}$ Nancy Kreiger présente une version écrite de son allocution de plénière intitulée « Epidemiology today: Mitigating threats to an ecosystem » («L'épidémiologie aujourd'hui : pour atténuer les menaces à un écosystème » [traduction libre]). Cette section spéciale est un moyen de mettre en relief la recherche épidémiologique canadienne dans la grande communauté de la santé publique.

Il n'existe pas au Canada de revue consacrée spécifiquement à l'épidémiologie. On en parle depuis au moins 20 ans, mais le lancement d'une revue canadienne d'épidémiologie n'est toujours pas une option viable. Si l'on compare les mandats des revues de santé publique et des revues d'épidémiologie, on remarque plus de similarités que de différences entre elles. La RCSP « se consacre à promouvoir l'excellence dans la recherche, les travaux d'érudition, les politiques et les pratiques de santé publique ", et l'American Journal of Public Health (AJPH) " se consacre à la publication de manuscrits originaux sur la recherche, les méthodes de recherche et l'évaluation de programmes dans le domaine de la santé publique » [traduction libre]. Comme d'autres revues d'épidémiologie, l'American Journal of Epidemiology (AJE) se décrit sur son site Web comme étant « consacré à la publication de résultats de recherches empiriques, d'articles d'opinions et de développements méthodologiques dans le domaine de la recherche épidémiologique " [traduction libre]. Ainsi, la plus grande différence semble être entre la « recherche en santé publique » et la " recherche épidémiologique ». On considère souvent l'épidémiologie comme « la science fondamentale de la santé publique », ce qui en fait une sous-catégorie de la recherche en santé publique. Elle met souvent davantage l'accent sur la recherche en amont, plutôt que sur la recherche appliquée, et sur les innovations méthodologiques. Mais au final, les deux disciplines ont le même objectif : améliorer la santé publique et des populations. L'épidémiologie a-t-elle donc un « port d'attache » à la RCSP? Absolument.

La Conférence biennale de la SCEB avait pour thème Paradigms to Pragmatism: Epidemiology and Biostatistics for the Changing World (« Des paradigmes au pragmatisme : l'épidémiologie et la biostatistique pour le monde qui change » [traduction libre]). Ce thème reflétait la vision d'une société professionnelle arrivée à maturité et célébrait un paysage exceptionnellement riche en contributions concrètes et méthodologiques au Canada. Les huit manuscrits publiés dans la section spéciale du présent numéro reflètent la diversité de la recherche épidémiologique qui était en 
conditions (dietary patterns and breast cancer risk, and risk factors for high school graduation and attention-deficit/hyperactivity disorder), one paper presents a review of the quality of administrative health databases in Canada, and three papers focus on development of novel methods for epidemiologic research. Although they are all "epidemiology" papers, they certainly fit within the purview of $\mathrm{CJPH}$ through fostering excellence in public health research and scholarship.

We are grateful to Dr. Nancy Kreiger for providing a written version of her plenary talk. She talks about the epidemiology ecosystem, which includes: scientists, its methods, the knowledge, colleagues and collaborators, funders and publishers. The health of this ecosystem depends on all of these parts and their interconnections. In recent years, epidemiologists have all experienced "threats" to their ecosystem, which as Dr. Kreiger points out can snowball and affect its precarious balance. The article, however, ends on an up-beat note, making suggestions to mitigate these threats and encouraging epidemiologists to work outside of the box.

We wished to secure an additional plenary paper to close the Special Section in this issue of CJPH. Dr. Sholom Wacholder proffered the opening keynote lecture in Toronto, in which he reflected on the perspective of using the proper set of tools to address substantive questions in molecular epidemiology. His depiction of epidemiology as the basic science of translational research resonated with the audience. His lecture was a roadmap for attaining relevance towards the noble goals of public health. Unbeknownst to most in the audience, Dr. Wacholder's delivery was made at great personal sacrifice, at the time enduring the pain of terminal cancer. He confided to us that he wished to return to Canada for one of his last professional engagements. His career had begun in Canada and he was very happy to have been called back to the country that had given him so much. He did not live long enough to send the written version of his magnificent lecture. However, we take the opportunity to honour Dr. Wacholder for his lifetime devotion to the epidemiology and public health communities, irrespective of national boundaries.

It is our hope that this Special Section becomes an ongoing collaboration between CJPH and CSEB. Future CSEB conferences will provide valuable scientific content for the pages of CJPH. We are grateful for the enthusiastic participation from both organizations and we welcome suggestions on how to expand or shape this collaboration in the future.

Linda Dodds

Senior Editor, CJPH, Dalhousie University, Halifax, NS

Eduardo Franco, Scientific Chair, CSEB Biennial Conference, 2015 Guest Editor, McGill University, Montreal, QC

doi: $10.17269 / \mathrm{CJPH} .107 .5460$ vitrine à Toronto en juin dernier. Deux des articles portent sur la répartition de résultats de santé (cancer du cerveau et temps consacré aux activités sédentaires), deux autres sur l'identification des déterminants et de l'étiologie de problèmes de santé (habitudes alimentaires et risque de cancer du sein, facteurs de risque de l'obtention du diplôme d'études secondaires et du trouble déficitaire de l'attention avec hyperactivité), un article examine la qualité des bases de données administratives sur la santé au Canada, et trois autres portent sur l'élaboration de nouvelles méthodes de recherche épidémiologique. Ce sont tous des articles " d'épidémiologie », mais ils s'inscrivent certainement dans le mandat de la RCSP du fait qu'ils favorisent l'excellence dans la recherche et l'érudition en santé publique.

Nous sommes reconnaissants à $\mathrm{M}^{\mathrm{me}}$ Nancy Kreiger de nous offrir une version écrite de son allocution de plénière. Elle y traite de l'écosystème de l'épidémiologie, qui comprend les scientifiques, les méthodes, les connaissances, les collègues et les collaborateurs, les bailleurs de fonds et les éditeurs. La santé de cet écosystème dépend de tous ces éléments et de leurs interconnexions. Depuis quelques années, les épidémiologistes sont aux prises avec des « menaces » à leur écosystème qui, comme le fait remarquer $\mathrm{M}^{\mathrm{me}}$ Kreiger, peuvent faire boule de neige et mettre en péril un équilibre précaire. L'article se termine néanmoins sur une note optimiste en faisant des suggestions pour atténuer ces menaces et en encourageant les épidémiologistes à sortir des sentiers battus.

Nous voulions une autre communication de plénière pour clore la section spéciale de ce numéro de la RCSP. M. Sholom Wacholder avait prononcé l'allocution d'ouverture de la conférence à Toronto; il y réfléchissait à l'utilisation d'un bon ensemble d'outils pour aborder les questions de fond en épidémiologie moléculaire. Son portrait de l'épidémiologie comme étant la science fondamentale de la recherche translationnelle avait trouvé écho auprès de l'auditoire. Son allocution était une feuille de route pour améliorer la pertinence à l'égard des nobles objectifs de la santé publique. À l'insu de la plupart des personnes présentes, M. Wacholder a prononcé cette allocution au prix d'un grand sacrifice personnel, car il souffrait d'un cancer terminal. Il nous a confié qu'il souhaitait retourner au Canada pour ce qui devait être l'un de ses derniers engagements professionnels. Sa carrière avait commencé au Canada, et il était très heureux d'avoir été rappelé dans ce pays qui lui avait tant donné. Il n'a pas survécu assez longtemps pour envoyer la version écrite de sa magnifique allocution. Nous profitons toutefois de l'occasion pour rendre hommage à Sholom Wacholder pour son dévouement de toute une vie à l'épidémiologie et aux communautés de la santé publique, indépendamment des frontières nationales.

Nous espérons que cette section spéciale deviendra une collaboration continue entre la RCSP et la SCEB. Les futures conférences de la SCEB fourniront de précieux contenus scientifiques pour les pages de la Revue. Nous sommes reconnaissants de la participation enthousiaste des deux organismes et nous accueillerons avec plaisir les suggestions sur les moyens d'élargir ou de faire évoluer cette collaboration à l'avenir

Linda Dodds, Rédactrice de la RCSP, Université Dalhousie, Halifax (Nouvelle-Écosse)

Eduardo Franco, Président scientifique, conférence bisannuelle SCEB, 2015; Rédacteur invité, Université McGill, Montréal (Québec)

doi: 10.17269/CJPH.107.5460 